\title{
ARTí́CULOS
}

Sometido 09.10.2014. Aprobado 24.03.2015

Este artículo tiene autoría de miembros del Cuerpo Editorial Científico de la RAE, se ha evaluado por el sistema double blind review, con exención y independencia. Editor Científico: Edmilson de Oliveira Lima

DOI: http://dx.doi.org/10.1590/So034-759020160105

\section{¿INFLUYE LA PERSONALIDAD DE LOS DIRECTIVOS DE PYMES EN LOS RESULTADOS EXPORTADORES?}

\author{
Does the personality of small and medium-sized companies' directors affect \\ exporting results?
}

\section{A personalidade dos diretores de pequenas e médias empresas influencia os resultados de exportação?}

\section{RESUMEN}

En este trabajo se analiza si los valores según la escala de Schwartz varían entre los directivos responsables de exportación de Pequeñas y Medianas Empresas (PyMEs) entre las empresas con mayor éxito exportador, frente a las que tienen un éxito exportador limitado. El estudio se aplica a una muestra de 196 PyMEs españolas exportadoras. En primer lugar se aplica un clúster de clases latentes para clasificar las empresas en función del performance exportador, para después aplicar test de comparaciones de medias y buscar diferencias significativas en los valores de Schwartz entre directivos de empresas de ambos grupos. Los valores en los que aparecen diferencias significativas son: honestidad, influencia, ambición, lealtad, justicia social, autoridad, vida excitante y orden social.

PALABRAS CLAVE | Performance exportador, escala de valores de Schwartz, internacionalización, Pequeñas y Medianas Empresas, clúster de clases latentes.

\begin{abstract}
In this work, we examine whether values in Schwartz's scale For Small and Medium-Sized Enterprises (SME) export managers from the most successful export companies differ from those for SME managers from companies with limited export success. The study was conducted with a sample of 196 Spanish export SMEs. First, we conducted a latent class cluster analysis to classify companies according to their export performance. Then, we employed means comparison test to find significant differences in Schwartz's scale between executives from companies in both groups. Significant differences were found for the following values: honesty, influence, ambition, loyalty, social justice, authority, exciting life, and social order.
\end{abstract}

KEYWORDS / Export performance, Schwartz value scale, internationalization, Small and Medium Enterprises, latent cluster analysis.

Profesor de la Universidad de Sevilla, Facultad de Ciencias Económicas y

Empresariales - Sevilla, España

\section{ANTONIO NAVARRO-GARCÍA}

anavarro@us.es

Profesor de la Universidad de Sevilla,

Facultad de Ciencias Económicas y

Empresariales - Sevilla, España

\section{JORGE ARENAS-GAITAN}

jarenas@us.es

Profesor de la Universidad de Sevilla,

Facultad de Ciencias Económicas y

Empresariales - Sevilla, España

\section{RESUMO}

No presente trabalho, examinamos se gerentes de exportação das Pequenas e Médias Empresas (PMEs) exportadoras mais bem-sucedidas apresentavam diferenças em valores na escala de Schwartz em comparação com gerentes de exportação de PMEs com sucesso limitado em exportações. $O$ estudo foi conduzido com uma amostra de 196 PMEs espanholas. Primeiramente, conduzimos uma análise de cluster de classes latentes para classificar as empresas segundo seu desempenho em exportações. Depois, empregamos um teste de comparação de médias para encontrar diferenças significativas em valores na escala de Schwartz entre executivos das empresas de ambos os grupos. Diferenças significativas foram encontradas para os seguintes valores: honestidade, influência, ambição, lealdade, justiça social, autoridade, vida excitante e ordem social.

PALAVRAS-CHAVE / Desempenho em exportações, escala de valores de Schwartz, internacionalização, Pequenas e Médias Empresas, análise de cluster latente. 


\section{INTRODUCCIÓN}

En cualquier situación empresarial saber distinguir los factores que llevan al éxito de una empresa es una cuestión capital. No menos importante, en una etapa de globalización de los mercados en la que nos encontramos, es analizar los factores que llevan al éxito de una empresa exportadora. Esto es así, ya que el número de empresas exportadoras en la mayoría de los países aumenta de forma progresiva. Si a esto unimos el hecho de investigar factores clave para el éxito exportador de Pequeñas y Medianas Empresas (PyMEs), teniendo en cuenta que las PyMEs suponen el $99 \%$ de las empresas de la OCDE, la justificación de cualquier estudio que permita mejorar su conocimiento y gestión es fundamental (AragónSánchez \& Rubio-Bañón, 2005). Su importancia en la creación de puestos de trabajo, innovación tecnológica y rejuvenecimiento económico está reconocido por todos los actores económicos, por ello, es importante que los factores que ayuden a explicar los resultados exportadores de las PyMEs sean una prioridad en la investigación (Freeman, Styles, \& Lawley, 2012).

Se puede definir la internacionalización de las PyMEs como un proceso de implicación creciente en operaciones internacionales, y este proceso suele ser gradual y secuencial, incluyendo varias etapas (Ruzzier, Hisrich, \& Antoncic, 2006).

En España según las “estadísticas PyME evolución e indicadores" de la Secretaría general de industria y de la pequeña y mediana empresa, el porcentaje de PyMEs respecto al total de empresas es del 99,88\% en el año 2013. El 6,54\% de las PyMEs son empresas del sector industrial, el $13,56 \%$ pertenecen a la construcción, el $24,32 \%$ al comercio y el $55,57 \%$ al sector servicios.

Además, diversos estudios refuerzan la idea de que las PyMEs internacionalizadas tienen mayor probabilidad de supervivencia (Floriani \& Fleury, 2012; Lee, Kelley, Lee, \& Lee, 2012), por lo que ayudar a éstas en su proceso de internacionalización implica mejorar su probabilidad de subsistencia a medio y largo plazo, tanto en economías desarrolladas como en economías en vías de desarrollo (Gashi, Hashi, \& Pugh, 2014).

Un aspecto clave de las PyMEs es la influencia de las características personales de sus directivos en la toma de decisiones. En las grandes empresas las decisiones tomadas en los equipos directivos son más consensuadas y corporativas. Sin embargo, en las PyMEs la influencia individual y el carácter de los directivos y/o propietarios tiene un mayor efecto en la toma de decisiones (Torre \& Solari, 2013), es decir, son organizaciones con menos trabas burocráticas, lo que da a los directivos más libertad de acción a la hora de tomar e implementar decisiones estratégicas (Håkonsson, Burton, Obel, \& Lauridsen, 2012). Por ejemplo, se ha demostrado el efecto del nivel educativo, edad o experiencia de los directivos en la toma de decisiones de
PyMEs (Wagner \& Paton, 2014). Respecto a la influencia de los directivos de PyMEs en la internacionalización, aspectos como la orientación global, experiencia de trabajo, conocimiento de lenguas extranjeras, o relaciones formales e informales de sus directivos son aspectos clave para una rápida internacionalización de sus empresas (Przybylska, 2013). Tampoco se pueden olvidar otras características de estos directivos tales como percepción del riesgo, tolerancia de la ambigüedad, resistencia al cambio y auto-confianza (Acedo \& Jones, 2007).

Sin embargo, junto a las características personales de los directivos, aunque la literatura también reconoce la importancia e influencia de los valores personales en la toma de decisiones y resultados empresariales (Hambrick, 2007), escaso es el conocimiento que se posee sobre esta cuestión, existiendo un gap investigador sobre el que es necesario ir avanzando. Con todo esto, el objetivo que nos planteamos en este trabajo es analizar si los valores, según la escala de Schwartz, varían entre los directivos responsables de exportación de PyMEs con mayor éxito exportador, frente a las que tienen un éxito exportador limitado. Por tanto, nuestra pregunta o cuestión de investigación es comprobar si las empresas con mayor éxito exportador tienen unos directivos con valores personales diferentes de aquellos que gestionan las exportaciones de empresas con un éxito exportador limitado.

Sobre la cuestión de investigación planteada, este trabajo tiene como principal contribución demostrar que efectivamente los directivos de PyMEs exportadoras con mejores resultados poseen un sistema de valores más proactivo frente a directivos de PyMEs exportadoras cuyo sistema de valores es más conservador.

Para lograr el objetivo planteado, el presente trabajo tiene la siguiente estructura. En primer lugar, se definen los antecedentes teóricos centrados en la internacionalización de las PyMEs, el resultado exportador y los sistemas de valores en los directivos. A continuación, se presenta la metodología empleada, definiéndose la muestra, las escalas de medidas y la herramienta de análisis de datos. Finalmente, se presentan los resultados y conclusiones del estudio, así como las limitaciones y futuras líneas de investigación.

\section{ANTECEDENTES TEÓRICOS}

En este apartado hacemos referencia a tres aspectos clave para entender el desarrollo teórico de este trabajo. Un primer subapartado dedicado a la internacionalización de las PyMEs, el segundo que trata sobre la medición de resultados exportadores, y el tercero que hace referencia a los valores personales de Schwartz aplicados a los directivos. 


\section{La internacionalización de las PyMEs}

La internacionalización de las PyMEs es un factor muy estudiado en la literatura reciente de negocios internacionales y marketing internacional. Los estudios se han centrado principalmente en las características distintivas de los procesos de internacionalización de este tipo de empresas, como son: rápidas expansiones internacionales, gran uso de redes y alianzas, expansión nacional e internacional al mismo tiempo, falta de recursos y experiencia y centrarse en nichos de mercados globales (Dickson, Weaver, \& Vozikis, 2013; Onkelinx, Manolova, \& Edelman, 2012; Schweizer, 2012). Según datos de la Unión Europea, un $25 \%$ de las PyMEs de la UE27 han hecho exportaciones en los tres años anteriores al estudio, que es el año 2009 (EU, 2009). Según este estudio las principales barreras que se encuentran las PyMEs para exportar son: (1) barreras internas como son el precio de su producto 0 servicio y alto coste de la internacionalización, y (2) barreras externas, tales como la falta de capital, falta de información adecuada, falta de apoyo institucional y los costes y dificultades burocráticas del transporte internacional.

Este mismo estudio concluye que las PyMEs internacionalizadas tienen mayores rendimientos económicos que las no internacionalizadas, junto con otras interesantes aportaciones como son: (1) mientras mayores son las PyMEs mayor es su probabilidad de exportar; (2) mientras más pequeño es el mercado doméstico también aumenta la probabilidad de exportar; (3) PyMEs fabricantes y mayoristas son especialmente activas en el comercio internacional; (4) las PyMEs más involucradas con la innovación de productos y procesos son más proclives al comercio internacional.

A partir de un estudio más reciente, también de la Unión Europea (Reinhard et al., 2013), se destaca que la mayoría de las agencias de promoción exterior europeas están fomentando la internacionalización hacia países del BRIC (Brasil, Rusia, India y China), así como USA y países limítrofes de la frontera europea como Turquía y Ucrania. Por tanto, Brasil se ha convertido en un objetivo prioritario para las PyMEs europeas internacionalizadas y muchas de estas empresas comenzarán su periplo por Sudamérica a partir de este país.

\section{Medición de resultados exportadores}

El resultado exportador es el output fundamental con el que se suele medir la actuación de la empresa exportadora en los mercados extranjeros y es un aspecto esencial para la toma de decisiones en el ámbito internacional (Madsen, 1998). Cavusgil y Zou definen el resultado exportador como la extensión por la cual la firma alcanza sus objetivos a través de la exportación de sus productos-marcas a los mercados extranjeros, incluyendo tanto aspectos económicos (beneficio, ventas, etc.) como estratégicos (posicionamiento internacional, incremento de la cuota de mercado derivado de la exportación, logro de objetivos internacionales, etc.), a través de la planificación y ejecución de la estrategia de marketing de la empresa exportadora (Cavusgil \& Zou, 1994).

Se han usado principalmente tres paradigmas alternativos para explicar teóricamente el performance exportador: paradigma performance-conducta-estructura, paradigma relacional y el enfoque basado en los recursos (Freeman et al., 2012). El primero, basado en la teoría de la organización industrial, indica que los resultados dependen principalmente de las características del sector en el que opera la empresa (Ruppenthal \& Bausch, 2009; Zou \& Cavusgil, 2002). El segundo, basado en la teoría de intercambio relacional, destaca la relevancia de la naturaleza e impacto de las relaciones exportador-cliente (Ural, 2009). El enfoque basado en los recursos (Resource-based view [RBV]) indica que las empresas pueden conseguir ventajas competitivas sostenibles distribuyendo recursos y capacidades valiosas que son inelásticas en el mercado (Akhter \& Robles, 2006). Algunos trabajos han integrado los tres enfoques con la idea de ofrecer un modelo integrador que reúna los diferentes determinantes del resultado exportador (Navarro, Acedo, Losada, \& Ruzo, 2011; Navarro-García, Rondán-Cataluña, \& Acedo-González, 2013). En todo caso, de la revisión de la literatura (Rose \& Shoham, 2002; Sousa, Martínez-López, \& Coelho, 2008) se desprende dos aspectos básicos del resultado exportador: (1) es un concepto multidimensional, el cual debe ser valorado a través de medidas cuantitativas (ventas, rentabilidad, crecimiento, etc.) y cualitativas (éxito percibido, satisfacción, logro de objetivos, etc.) (Freeman et al., 2012); (2) las medidas empleadas para su evaluación deben reflejar las percepciones directivas sobre los resultados alcanzados (ej.: satisfacción directiva con el resultado exportador) (Lages, Jap, \& Griffith, 2008).

En el presente trabajo se tienen en cuenta los dos aspectos señalados (1) se conciben dos dimensiones del resultado exportador, una cuantitativa -crecimiento de las ventas de exportación y rentabilidad de las exportaciones - y otra cualitativa -satisfacción directiva-; (2) se tienen en cuenta las percepciones directivas (satisfacción directiva global) asociada a la actividad exportadora.

\section{Los valores de Schwartz en los directivos}

Los valores personales son los principios que de una forma estable se emplean para evaluar el comportamiento propio y el de los demás. Generan una forma subjetiva de interpretar la 
realidad asociada al entorno en el que se actúa (Finkelstein, Hambrick, \& Cannella, 2008). La teoría de valores básicos humanos de Schwartz (Schwartz, 1992) se ha utilizado con gran profusión durante más de 20 años en estudios de diversas áreas de conocimiento, países y con objetivos muy diversos (Schwartz et al., 2012). Hay varios factores que diferencian la escala de valores de Schwartz de otras anteriores, destacando la universalidad de la estructura y del contenido de esos valores. Así los 10 tipos de valores propuestos por su teoría son valores reconocidos en todas las culturas. En la Tabla 1 se presentan la definición de cada uno de los valores y los valores representativos de los mismos.

\section{Tabla 1. Ítems y definición de la escala de valores de Schwartz}

\begin{tabular}{|c|c|c|}
\hline Tipo & Definición & Valores representativos \\
\hline Estimulación & Excitación, novedad y desafío en la vida & Osadía, una vida variada, una vida excitante \\
\hline Hedonismo & Placer y gratificación sensorial para uno mismo & Placer, vida placentera \\
\hline Poder & $\begin{array}{l}\text { Estatus social y prestigio, control o dominios de las } \\
\text { personas o recursos }\end{array}$ & Poder social, autoridad, riqueza \\
\hline Seguridad & $\begin{array}{l}\text { Seguridad, armonía, y estabilidad de la sociedad, de } \\
\text { las relaciones sociales y de uno mismo }\end{array}$ & $\begin{array}{l}\text { Seguridad familia, seguridad nacional, orden social, } \\
\text { decencia, reciprocidad en los favores }\end{array}$ \\
\hline Tradición & $\begin{array}{l}\text { Respeto, compromiso y aceptación de las costumbres } \\
\text { e ideas que la cultura tradicional o religión brindan }\end{array}$ & $\begin{array}{l}\text { Aceptar ser parte de la vida, humildad, devoción } \\
\text { respecto por la tradición, moderación }\end{array}$ \\
\hline Benevolencia & $\begin{array}{l}\text { Preservación y bienestar de la gente con quien uno } \\
\text { está en frecuente contacto personal }\end{array}$ & $\begin{array}{l}\text { Amabilidad, honestidad, perdón, lealtad, } \\
\text { responsabilidad }\end{array}$ \\
\hline Universalismo & $\begin{array}{l}\text { Entendimiento, apreciación, tolerancia, y protección } \\
\text { del bienestar de todas personas y de la naturaleza }\end{array}$ & $\begin{array}{l}\text { Tolerancia, sabiduría, justicia social, equidad, un } \\
\text { mundo en paz, un mundo de belleza, unidad con la } \\
\text { naturaleza, protección del ambiente }\end{array}$ \\
\hline
\end{tabular}

Un aspecto interesante respecto a estos valores culturales es el alto nivel de consenso con respecto a la importancia relativa de los 10 valores citados entre diferentes sociedades. En la gran mayoría de las naciones estudiadas benevolencia, universalismo y auto-dirección son los aspectos más relevantes y tradición, poder y estimulación son los menos. Esto indica que aspectos de la naturaleza humana y del funcionamiento social que conforman las prioridades individuales de valores son ampliamente compartidas entre culturas diversas (Schwartz et al., 2012).

Con respecto a los directivos, estos siguen modelos psicológicos que influyen en la forma de percibir el entorno y afectan a su toma de decisiones (Johnson \& Hoopes, 2003; Nadkarni \& Barr, 2008). Las creencias y valores individuales ejercen una influencia importante en la creación de las opiniones y modelos mentales de los directivos (Hambrick, 2007; Soriano \& Castrogiovanni, 2012). En este contexto, es importante comprender el papel que pueden jugar los valores personales en la toma de decisiones empresariales, pues influye en la forma en que los directivos interpretan las situaciones de mercado, las decisiones estratégicas adoptadas, los recursos comprometidos, etc., y, por tanto, pueden ser determinantes de los resultados empresariales (White, Varadarajan, \& Dacin, 2003).

Desde el punto del continuo que supone el inventario de valores humanos (Schwartz, 1992), dos posturas cabe destacar desde la óptica directiva: (a) enfoque conservador, caracterizado por un predominio de la visión individualista, de mantenimiento del status quo y que se muestra reacia a realizar cambios, en la cual predominan los valores de seguridad, conformidad, tradición, poder y logro. Esta postura implica aplicar una lógica tradicional, fruto de una cultura reactiva, en la resolución de problemas, toma de decisiones, actitudes y comportamientos empresariales (Schwartz \& Bardi, 2001). Sus repercusiones 
suelen ser negativas en la rapidez de respuesta y adaptación a los deseos de los consumidores, motivaciones para innovar, o diversificar mercados (Krasnikov \& Jayachandran, 2008); (b) enfoque proactivo, caracterizado por un predominio de la visión colectiva, de apertura al cambio y predisposición para realizar adaptaciones y orientarse al mercado, donde predominan los valores de estimulación, independencia de pensamiento o autodirección, hedonismo, benevolencia y universalismo (Sousa, Ruzo, \& Losada, 2010).

Algunos estudios ha evidenciado los planteamientos expuestos (Carpenter, Geletkanycz, \& Sanders, 2004; Hambrick, 2007). Sin embargo, en el ámbito exportador, como señalan Sousa et al. (Sousa et al., 2010)) existe un importante desconocimiento del papel que desempeñan los valores de los responsables de exportación en las decisiones adoptadas y éxito alcanzado por la empresa exportadora en los mercados exteriores, a pesar de que algunos trabajos han mostrado la gran relevancia de su estudio (Leonidou, Katsikeas, \& Piercy, 1998). En este gap investigador encuentra su fundamento el presente trabajo, describiendo a continuación la metodología desarrollada para alcanzar los objetivos propuestos.

\section{METODOLOGÍA}

Esta sección se divide en la explicación de la muestra de empresas obtenidas, después se analizarán las escalas de medida utilizadas y como tercer subapartado se exponen los análisis estadísticos llevados a cabo.

\section{Muestra}

La muestra de empresas españolas obtenidas se obtuvo a partir de la base de datos de empresas exportadoras del Instituto de Comercio Exterior (ICEX). Se mantuvo la proporcionalidad sectorial, los cuestionarios se enviaron por e-mail a 1200 directivos responsables de las exportaciones de sus empresas. Se obtuvieron 196 encuestas de empresas con menos de 250 empleados (PyMEs). Se obtuvo una tasa de respuesta del 16,3\%, que está dentro de los límites normales de respuesta a encuestas mandadas por e-mail.

En cada una de las empresas exportadoras, se seleccionó un informante único para que completara el cuestionario. El empleo de un informante único reduce los errores y sesgos asociados a la tenencia de diferentes perspectivas de una misma cosa, ocurriendo cuando se emplea más de un informante en cada empresa (Huber \& Power, 1985). Para asegurar la fiabilidad de la fuente de información, se pidió al máximo responsable de la actividad exportadora que completase el cuestionario. En este sentido, el cuestionario incluía una sección específica donde se le preguntaba al encuestado por diversas características personales, entre las que se incluía el tipo de responsabilidad asociado a la actividad exportadora, evitando los sesgos asociados al posible desconocimiento de las cuestiones planteadas.

\section{Escalas de medida}

Para medir la escala de valores de Schwartz se ha seguido el “Draft Users Manual”, que el mismo Prof. Shalom Schwartz publicó (Schwartz, 2009) con el fin de ayudar a los investigadores que utilicen su escala para usarla correctamente. La escala se tradujo al castellano para pasarla en el cuestionario. Los 45 ítems de la escala SVS han demostrado tener una fuerte fiabilidad entre distintas culturas (Schwartz, 1992). Los valores y sus definiciones se presentan en la Tabla 1 . Se midieron con una escala métrica propuesta por Schwartz desde -1 a 7. Donde el -1 representa que el ítem tiene un significado opuesto o contrario a los valores del encuestado, o, significa que ese valor no es nada importante para el encuestado, y así sucesivamente hasta 7 que significa que dicho valor es de suprema importancia para el entrevistado.

Respecto al resultado exportador, se ha adoptado una visión formativa del constructo, al estar compuesto por dimensiones de naturaleza muy diferente (crecimiento de las ventas, rentabilidad y satisfacción global directiva). Desde esta perspectiva, siguiendo a algunos autores (Cadogan, Diamantopoulos, \& Siguaw, 2002; Huber \& Power, 1985), la dimensión cualitativa del resultado exportador fue evaluada a través de la satisfacción global percibida (1.- Totalmente insatisfecho...7.- Totalmente satisfecho) por los responsables de exportación asociado a los resultados de la actividad exportadora. La dimensión cuantitativa fue medida a través del crecimiento de las ventas y de la rentabilidad derivadas de la exportación en el último año (Cavusgil \& Zou, 1994; Navarro et al., 2011).

\section{Análisis estadísticos}

Teniendo en cuenta que la cuestión o pregunta de investigación de este trabajo es analizar si los valores, según la escala de Schwartz, varían entre los directivos responsables de exportación de PyMEs entre las empresas con mayor éxito exportador, frente a las que tienen un éxito exportador limitado, el primer paso es clasificar 
a las empresas de la muestra en función de sus resultados exportadores. Para ello hemos utilizado un clúster de clases latentes usando las variables sobre performance exportador que estaban recogidas en el cuestionario que son: satisfacción global con las exportaciones, crecimiento de las ventas de exportación el último año y rentabilidad de las exportaciones en el último año. Otros trabajos han demostrado las ventajas del clúster de clases latentes, frente a las técnicas tradicionales de conglomerados (Rondan-Cataluna, Sanchez-Franco, \& Villarejo-Ramos, 2010) entre las que destacan: creación de segmentos a posteriori, se pueden usar variables cualitativas, y los casos se asignan a cada grupo en función de sus probabilidades de pertenencia que se estiman directamente del modelo.

Una vez clasificadas las empresas en grupos usamos test t de comparación de medias, y como los datos no cumplen el requisito de normalidad usamos también los test no paramétricos siguientes: prueba de la mediana para muestras independientes, U de Mann-Whitney y la prueba de Kolmogorov-Smirnov para muestras independientes. En este caso, analizamos cada uno de los ítems de la escala de valores de Schwartz de los directivos de las PyMEs de la muestra para cada uno de los grupos de empresas obtenidos del clúster de clases latentes, viendo de esta manera si hay diferencias significativas entre ellos.

\section{RESULTADOS}

Como se indica en la sección anterior, primero hicimos un clúster de clases latentes usando las variables de performance exportador, atendiendo al criterio de menor BIC (Bayesian Information Criterion), nos quedamos con el modelo de dos grupos o clases latentes (ver Tabla 2).

\section{Tabla 2.Selección del número de clústeres}

\begin{tabular}{l|l|c|c|c|c|c|c|c}
\hline & & LL & BIC(LL) & Npar & L2 & df & p-value & Class.Err. \\
\hline Model1 & 1-Clúster & $-737,5576$ & 1538,4526 & 12 & 129,9805 & 92 & 0,0056 & 0 \\
\hline Model2 & 2-Clúster & $-711,2792$ & 1507,0083 & 16 & 77,4238 & 88 & 0,78 & 0,1033 \\
\hline Model3 & 3-Clúster & $-703,817$ & 1513,1963 & 20 & 62,4993 & 84 & 0,96 & 0,1068 \\
\hline Model4 & 4-Clúster & $-702,2803$ & 1531,2353 & 24 & 59,4259 & 80 & 0,96 & 0,2288 \\
\hline Model5 & 5-Clúster & $-702,3152$ & 1552,4177 & 28 & 59,4958 & 76 & 0,92 & 0,3313 \\
\hline Model6 & 6-Clúster & $-700,8099$ & 1570,5195 & 32 & 56,4852 & 72 & 0,91 & 0,3789 \\
\hline
\end{tabular}

Respecto a las medidas de bondad de ajuste, el modelo con dos grupos tiene un error de clasificación de $0.103, \mathrm{R}^{2}$ de entropía de $0.617, \mathrm{R}^{2}$ standard de 0.657 , valores todos ellos aceptables.

Los dos clústeres que salen son los siguientes:

\section{Tabla 3. Descripción de los clústeres}

\begin{tabular}{l|c|c}
\hline & Clúster1 & Clúster2 \\
\hline Tamaño Clúster & 0,7071 & 0,2929 \\
\hline Indicadores & & \\
\hline SAT. GLOBAL EXPORT. & 3,791 & 2,658 \\
\hline Media & 5,0252 & 2,5175 \\
\hline CREC. VENTAS EXPORT. ULT. AÑO & \\
\hline Media & 2,8918 & 2,3902 \\
\hline RENTAB. EXPORT ULT. AÑO &
\end{tabular}

Se observa en la Tabla 3 que el clúster 1 engloba a casi el $71 \%$ de las empresas de la muestra, y el segundo algo más del $29 \%$. El clúster 1 representa a las empresas con mayor éxito exportador ya que la satisfacción global con las exportaciones, el crecimiento de las ventas de exportación y la rentabilidad de las exportaciones del último año están valorados mucho más positivamente que en el clúster 2 , que está formado por aquellas empresas cuyos directivos perciben un desempeño exportador limitado.

El siguiente paso fue depurar la muestra, eliminando los casos en los que no se habían rellenado por parte del responsable de exportación la parte de la encuesta relacionada con los valores de Schwartz. No se eliminaron en la fase anterior porque de estas empresas si teníamos datos de resultados. Se pasó de 196 a 183 PyMEs exportadoras.

Las empresas son asignadas al clúster al que tienen mayor probabilidad de inclusión. Así aparecen 127 en el clúster 1 y 56 en el clúster 2 . Aplicamos un test t de comparación de medias, y como los datos no cumplen el requisito de normalidad usamos también los test no paramétricos siguientes: prueba de la mediana para muestras independientes, $U$ de Mann-Whitney y la prueba de Kolmogorov-Smirnov para muestras independientes. La Tabla 4 refleja sólo las variables que en el test t y en alguno de los no paramétricos mostraron diferencias significativas entre los dos grupos son los que hemos señalado, buscando al máximo la robustez de los resultados obtenidos. 
Tabla 4. Prueba t para la igualdad de medias

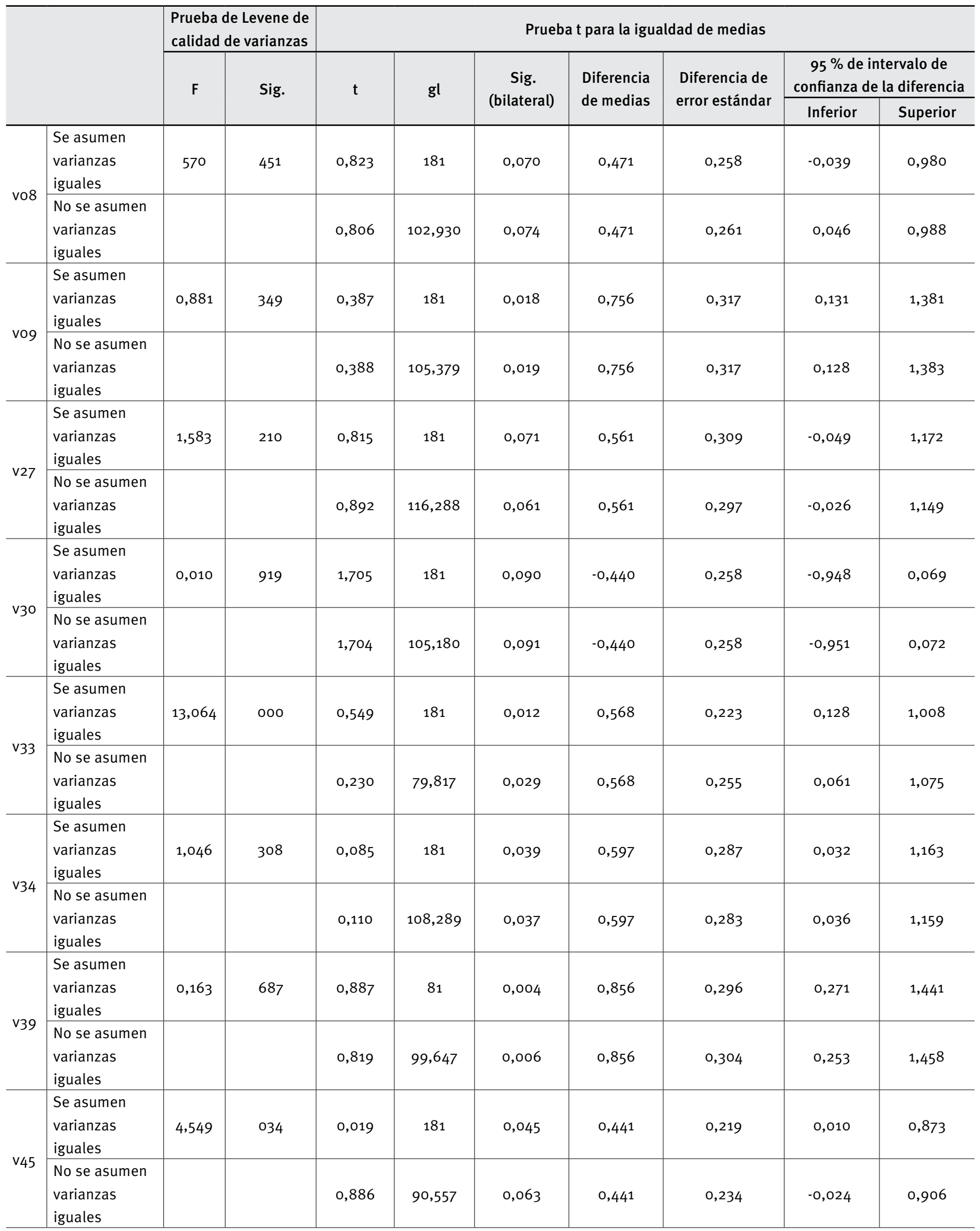


Los ítems 8, 9, 27, 30, 33, 34, 39 y 45 de la escala de Schwartz muestran diferencias significativas entre los directivos de las empresas del clúster 1 y el clúster 2 , con un nivel de confianza de al menos el $90 \%$. Analizamos para estos ítems los test no paramétricos de diferencias de medias para muestras independientes (Tabla 5).

Tabla 5. Pruebas no paramétricas para muestras independientes

\begin{tabular}{c|c|c|c}
\hline $\begin{array}{c}\text { Ítems de la escala } \\
\text { de Schwartz }\end{array}$ & $\begin{array}{c}\text { Significación asintótica de la } \\
\text { Prueba de la mediana para muestras } \\
\text { independientes }\end{array}$ & $\begin{array}{c}\text { Significación asintótica de la U de } \\
\text { Mann-Whitney }\end{array}$ & $\begin{array}{c}\text { Significación asintótica de } \\
\text { Kolmogorov- Smirnov para muestras } \\
\text { independientes }\end{array}$ \\
\hline V08 & 0,063 & 0,044 & 0,068 \\
\hline V09 & 0,007 & 0,012 & 0,033 \\
\hline$V_{27}$ & 0,078 & 0,071 & 0,35 \\
\hline$V_{30}$ & 0,066 & 0,071 & 0,156 \\
\hline$V_{33}$ & 0,588 & 0,037 & 0,033 \\
\hline$V_{34}$ & 0,007 & 0,008 & 0,177 \\
\hline$V_{39}$ & 0,114 & 0,086 & 0,468 \\
\hline
\end{tabular}

Todos estos ítems de la escala de Schwartz han mostrado diferencias significativas entre los directivos de PyMEs exportadoras en alguno de los test no paramétricos y en el test t de igualdad de medias con un nivel de confianza de al menos $90 \%$. A continuación, se presentan los valores medios de dichos ítems en cada uno de los clústeres (Tabla 6).

Tabla 6. Estadísticas de grupo

\begin{tabular}{|c|c|c|c|c|c|}
\hline & $\begin{array}{l}\text { Clúster } \\
\text { modal }\end{array}$ & $\mathrm{N}$ & Media & $\begin{array}{l}\text { Desviación } \\
\text { estándar }\end{array}$ & $\begin{array}{c}\text { Media de error } \\
\text { estándar }\end{array}$ \\
\hline \multirow{2}{*}{ vo8 } & 1 & 127 & 4,69 & 1,597 & 0,142 \\
\hline & 2 & 56 & 4,21 & 1,637 & 0,219 \\
\hline \multirow{2}{*}{ vo9 } & 1 & 127 & 3,76 & 1,975 & 0,175 \\
\hline & 2 & 56 & 3,00 & 1,973 & 0,264 \\
\hline \multirow{2}{*}{ v27 } & 1 & 127 & 3,28 & 1,987 & 0,176 \\
\hline & 2 & 56 & 2,71 & 1,786 & 0,239 \\
\hline \multirow{2}{*}{ v30 } & 1 & 127 & 4,88 & 1,607 & 0,143 \\
\hline & 2 & 56 & 5,32 & 1,608 & 0,215 \\
\hline \multirow{2}{*}{ v33 } & 1 & 127 & 5,69 & 1,212 & 0,108 \\
\hline & 2 & 56 & 5,13 & 1,727 & 0,231 \\
\hline \multirow{2}{*}{ v34 } & 1 & 127 & 4,47 & 1,803 & 0,160 \\
\hline & 2 & 56 & 3,88 & 1,748 & 0,234 \\
\hline \multirow{2}{*}{ v39 } & 1 & 127 & 3,68 & 1,812 & 0,161 \\
\hline & 2 & 56 & 2,82 & 1,927 & 0,257 \\
\hline \multirow{2}{*}{ V45 } & 1 & 127 & 5,64 & 1,283 & 0,114 \\
\hline & 2 & 56 & 5,20 & 1,531 & 0,205 \\
\hline
\end{tabular}

Así los ítems de la escala SVS que muestran diferencias significativas entre los directivos encargados de las exportaciones en PYMES entre los clústeres 1 (éxito exportador) y 2 (poco éxito exportador) son: 8 orden social, 9 una vida excitante, 27 autoridad, 30 justicia social, 33 lealtad, 34 ambición, 39 influencias, 45 honestidad. Por tanto, encontramos diferencias significativas en 6 de las 10 dimensiones de la escala: seguridad, estimulación, poder, universalismo, benevolencia y logro.

\section{DISCUSIÓN}

La mayoría de estudios centrados en el análisis de la influencia de los aspectos personales de los directivos en la toma de decisiones y resultados empresariales en el contexto internacional se ha centrado en las propias características personales (Przybylska, 2013; Wagner \& Paton, 2014) y pocos trabajos han ofrecido conclusiones sobre la repercusión de los valores. En este trabajo se demuestra algo que intuitivamente se podría pensar, pero que es difícil de demostrar, y es que los directivos dedicados a la exportación de las PyMEs con mayor éxito exportador poseen valores y rasgos de personalidad que difieren significativamente de los directivos de PyMEs cuyos resultados exportadores no son tan buenos. 
En particular los aspectos que difieren son los siguientes. Para los directivos de PyMEs con éxito exportador es más importante el orden social y la estabilidad de la sociedad que para sus colegas de PyMEs con menor resultado exportador. Es decir, este aspecto de la seguridad es una dimensión que predomina en los directivos de empresas exportadoras con mejores resultados en los mercados extranjeros. Esto nos indica que los directivos con PyMEs exportadoras de mayor éxito tenderán a buscar mercados más estables, con menores posibilidades de problemas sociales, revueltas o desorden público. Así pues, estos directivos evitarán dentro de lo posible las aventuras internacionales en países con poca estabilidad política y social (Cadogan, Cui, \& Li, 2003).

También, los primeros le dan mayor importancia a tener una vida excitante y llena de experiencias estimulantes, aflorando la dimensión de estimulación. La internacionalización en una PYME siempre es un reto estimulante para sus directivos, cuyas empresas en muchas ocasiones no se han enfrentado anteriormente a esta actividad y por tanto, suponen retos profesionales y personales importantes. Como consecuencia, estos valores personales de preferencia de una vida estimulante y excitante les llevan a tener una visión más proactiva respecto a la internacionalización.

Estos directivos tienden a confiar más en su autoridad y dirigir o capitanear equipos de personas, reflejando una capacidad de poder. Esto implica que ese afán de liderazgo, de ejercer poder y autoridad les lleva a ser personas con más confianza en sí mismos, y quizás por ello con menor aversión al riesgo y más proclives a aventuras internacionales de éxito.

Por otra parte los directivos de PyMEs exportadoras de éxito suelen ser más leales a las personas y a los grupos, a ser más sinceros y honestos, aspectos estos relacionados con la benevolencia. Es decir, lealtad, honestidad y sinceridad son valores personales importantes para estos individuos, estas son cuestiones muy intrincadas con el marketing relacional. Estos valores suelen influir en mejorar las relaciones con otras organizaciones que permitan mantener relaciones comerciales duraderas, estables y exitosas. Todos estos valores son claves para el desarrollo y mantenimiento de canales de distribución internacionales (Saleh, Ali, \& Mavondo, 2014).

Otro valor a destacar por los directivos de PyMEs internacionalizadas con éxito es ser ambiciosos y trabajar duro para aspirar a más logros y a ser más influyente teniendo más impacto en las personas y acontecimientos estos son aspectos asociados al logro. Para estas personas la ambición de conseguir mejores resultados, la capacidad para trabajar duro y el incentivo de tener más influencia en el mercado son elementos clave en su personalidad. Y según nuestros resultados es otro de los aspectos que los distingue de directivos con peores resultados exportadores.

Todos estos valores muestran el predominio de un sistema de valores proactivo, caracterizado por una visión colectiva de los negocios, de tolerancia y apertura al cambio, y predisposición para realizar adaptaciones y orientarse al mercado, repercutiendo positivamente en el resultado exportador (Sousa et al., 2008; Sousa et al., 2010).

Sin embargo, para los directivos de PyMEs con éxito exportador es menos importante corregir las injusticias o cuidar del débil que para los directivos del grupo de PyMEs con menos éxito exportador, este aspecto está relacionado con el universalismo, mostrándose éstos últimos más proteccionistas o conservadores. Este aspecto nos indica que en los negocios internacionales esos valores más altruistas, más empáticos que se centran más en ayudar al prójimo están más vinculados con directivos de empresas con un éxito exportador limitado. Es decir, en la jungla de los negocios el pensar en los débiles puede ser contraproducente en términos de resultados empresariales.

Ello puede ser un reflejo de un enfoque conservador, caracterizado por un predominio de la visión individualista, de mantenimiento del status quo, mostrándose reacio a realizar cambios. Esta postura implica aplicar una lógica dominante tradicional, fruto de una cultura reactiva, en la resolución de problemas, toma de decisiones, actitudes y comportamientos empresariales (Schwartz \& Bardi, 2001). Sus repercusiones suelen ser negativas en la rapidez de respuesta y adaptación a los deseos de los consumidores, motivaciones para innovar, o diversificar mercados (Krasnikov \& Jayachandran, 2008). Todo ello puede repercutir negativamente en los resultados empresariales, como se refleja en la presente investigación.

Es interesante resaltar el hecho de que corregir las injusticias o cuidar del débil, sea el único valor en los que hemos encontrado diferencias significativas al que los directivos de PyMEs con menos éxito exportador han dado más importancia que a los directivos del otro grupo. Parece que estos aspectos más sociales, más asertivos, tienen menos presencia en los directivos de PyMEs con éxito exportador. El perfil de estos directivos es más agresivo en los que una vida excitante, la autoridad, la lealtad, el orden social, ambición, ser influyente y sincero son valores a los que les dan más importancia que sus colegas de PyMEs con menor éxito exportador.

\section{CONCLUSIONES}

En definitiva, este trabajo demuestra que las empresas con mayor éxito exportador tienen unos directivos con valores personales 
diferenciados de aquellos que gestionan las exportaciones de empresas con un éxito exportador limitado. Este hecho que puede parecer obvio no estaba justificado de manera científica y puede ser muy importante a la hora de reclutar personal que se encargue de las labores de internacionalización de las PyMEs.

Hemos encontrado un perfil de directivo de PyMEs internacionalizadas con éxito con unas características personales muy marcadas entre las que destacan: (1) la importancia del orden social y la estabilidad de la sociedad; (2) tienen preferencia por una vida estimulante y excitante; (3) confían más en su autoridad y en dirigir equipos de personas; (4) lealtad, honestidad y sinceridad son valores personales importantes para estos individuos; (5) por otra parte, ambición, trabajar duro para aspirar a más logros y ser más influyentes son características muy valoradas por ellos; (6) y por último, para ellos es menos importante corregir las injusticias o cuidar del débil que para los directivos del grupo de PyMEs con menos éxito exportador.

Para finalizar, señalar la principal limitación de este trabajo como es considerar exclusivamente la influencia de los valores en los resultados empresariales, obviando otros aspectos sobre los que la literatura apunta una repercusión más directa como son las orientaciones directivas (Escandon, Vargas, \& González-Campo, 2013), decisiones estratégicas (Hagen, Zucchella, Cerchiello, \& Giovanni, 2012) y alianzas estratégicas (Nakos, Brouthers, \& Dimitratos, 2014). Actuando estos factores, en ocasiones, como mediadores o moderadores de la relación valores-resultados empresariales. Por ejemplo, se ha demostrado la influencia positiva de la inversión en nuevos productos y contratar a personal ajeno a la familia de los propietarios como factores que influyen positivamente en los resultados exportadores de las empresas (D'angelo, Majocchi, Zucchella, \& Buck, 2013). Esta limitación constituye una guía para orientar futuros trabajos de investigación. También señalar que este trabajo se ha centrado en los valores de una única persona por empresa, el responsable de exportación o export manager, el cual aun asumiendo que representa el sistema de valores de la empresa exportadora, no tiene por qué ser exactamente así, siendo conveniente en futuros trabajos tener en cuenta los valores de otros directivos o personas que desarrollan funciones asociadas a la actividad exportadora, lo cual ofrecerá una perspectiva más enriquecedora de la relación valores directivos-resultados de la exportación. Además, sería recomendable conocer los valores personales de directivos de distintos de PyMEs internacionalizadas de países culturalmente diferenciados puede ser muy interesante y se podría relacionar con toda la literatura de internacionalización y análisis crosscultural (Hofstede, 2001; Schwartz \& Bardi, 2001).

\section{Nota de los autores}

Investigación financiada con el proyecto de Excelencia de la Junta de Andalucía (España) con referencia: P11SEJ-7042

\section{REFERENCIAS}

Acedo, F. J., \& Jones, M. V. (2007). Speed of internationalization and entrepreneurial cognition: Insights and a comparison between international new ventures, exporters and domestic firms. Journal of World Business, 42(3), 236-252. doi:10.1016/j.jwb.2007.04.012

Akhter, S. H., \& Robles, F. (2006). Leveraging internal competency and managing environmental uncertainty: Propensity to collaborate in international markets. International Marketing Review, 23(1), 98-115. doi:10.1108/02651330610646313

Aragón-Sánchez, A., \& Rubio-Bañón, A. (2005). Factores asociados con el éxito competitivo de las pyme industriales en España. Universia Business Review. Retrieved from https://ubr.universia.net/

Cadogan, J. W., Cui, C. C., \& Li, E. K. Y. (2003). Export market-oriented behavior and export performance: The moderating roles of competitive intensity and technological turbulence. International Marketing Review, 20(5), 493-513. doi:10.1108/02651330310498753

Cadogan, J. W., Diamantopoulos, A., \& Siguaw, J. A. (2002). Export market-oriented activities: Their antecedents and performance consequences. Journal of International Business Studies, 33(3)615-626.

Carpenter, M. A., Geletkanycz, M. A., \& Sanders, W. G. (2004). Upper echelons research revisited: Antecedents, elements, and consequences of top management team composition. 30(6), 749-778. doi:10.1016/j.jm.2004.06.001

Cavusgil, S. T., \& Zou, S. (1994). Marketing strategy-performance relationship: An investigation of the empirical link in export market ventures. Journal of Marketing, 58(1), 1-21. doi:10.2307/1252247

D’Angelo, A., Majocchi, A., Zucchella, A., \& Buck, T. (2013). Geographical pathways for SME internationalization: Insights from an Italian sample. International Marketing Review, 30(2), 80-105. doi:10.1108/02651331311314538

Dickson, P. H., Weaver, K. M., \& Vozikis, G. S. (2013). The impact of the institutional environment on SME internationalization: An assessment of the environmental assumptions of emerging integrated models of internationalization. Journal of Applied Business and Economics, 15(3), 43-55.

Escandon, B. D. M., Vargas, G. M., \& González-Campo, C. H. (2013). Determinantes de los resultados internacionales de las PyMEs de reciente creación en Colombia, 2011. Revista Innovar, 23(49), 17-30.

Finkelstein, S., Hambrick, D. C., \& Cannella, A. A., Jr. (2008). Strategic leadership: Theory and research on executives, top management teams, and boards. New York: Oxford University Press.

Floriani, D. E., \& Fleury, M. T. (2012). O efeito do grau de internacionalização nas competências internacionais e no desempenho financeiro da PME brasileira. RAC-Revista de Administração Contemporânea, 16(3), 438-458. doi:10.159o/s1415-65552012000300007

Freeman, J., Styles, C., \& Lawley, M. (2012). Does firm location make a difference to the export performance of SMEs? International Marketing Review, 29(1), 88-113. doi:10.1108/02651331211201552 
Gashi, P., Hashi, I., \& Pugh, G. (2014, February). Export behaviour of SMEs in transition countries. Small Business Economics, 42(2), 407435. doi:10.1007/s11187-013-9487-7

Hagen, B., Zucchella, A., Cerchiello, P., \& Giovanni, N. (2012). International strategy and performance-clustering strategic types of SMEs. International Business Review, 21(3), 369-382. doi:10.1016/j.ibusrev.2011.04.002

Håkonsson, D. D., Burton, R. M., Obel, B., \& Lauridsen, J. (2012). Strategy implementation requires the right executive style: Evidence from Danish SMEs. Long Range Planning, 45(2-3), 182-208. doi:10.1016/j. Irp.2012.02.004

Hambrick, D. C. (2007). Upper echelons theory: An update. Academy of Management Review, 32(2), 334-343. doi:10.2307/20159303

Hofstede, G. H. (2001). Culture's consequences: Comparing values, behaviors, institutions and organizations across nations. London: Sage.

Huber, G. P., \& Power, D. J. (1985). Retrospective reports of strategic level managers: Guidelines for increasing their accuracy. Strategic Management Journal, 6(2), 171-180. doi:10.1002/smj.4250060206

Johnson, D. R., \& Hoopes, D. G. (2003). Managerial cognition, sunk costs, and the evolution of industry structure. Strategic Management Journal, 24(10), 1057-1068. doi:10.1002/smj.320

Krasnikov, A., \& Jayachandran, S. (2008). The relative impact of marketing, research-and-development, and operations capabilities on firm performance. Journal of Marketing, 72(4), 1-11. doi:10.1509/ jmkg.72.4.1

Lages, L. F., Jap, S. D., \& Griffith, D. A. (2008). The role of past performance in export ventures: A short-term reactive approach. Journal of International Business Studies, 39(2), 304-325. doi:10.1057/palgrave.jibs. 8400339

Lee, H., Kelley, D., Lee, J., \& Lee, S. (2012). SME survival: The impact of internationalization, technology resources, and alliances. Journal of Small Business Management, 50(1), 1-19. doi:10.1111/j.1540627X.2011.00341.X

Leonidou, L. C., Katsikeas, C. S., \& Piercy, N. F. (1998). Identifying managerial influences on exporting: Past research and future directions. Journal of International Marketing, 6(2), 74-102.

Madsen, T. K. (1998). Managerial judgment of export performance. Journal of International Marketing, 6(3), 82-93.

Ruzzier, M., Hisrich, R., \& Antoncic, B. (2006). SME internationalization research: Past, present, and future. Journal of SmaIl Business and Enterprise Development, 13(4), 476-497. doi:10.1108/14626000610705705

Nadkarni, S., \& Barr, P. S. (2008). Environmental context, managerial cognition, and strategic action: An integrated view. Strategic Management Journal, 29(13), 1395-1427. doi:10.1002/smj.717

Nakos, G., Brouthers, K. D., \& Dimitratos, P. (2014. International alliances with competitors and non-competitors: The disparate impact on SME international performance. Strategic Entrepreneurship Journal, 8(2), 167-182. doi: $10.1002 /$ sej.1169

Navarro, A., Acedo, F. J., Losada, F., \& Ruzo, E. (2011). Integrated model of export activity: Analysis of heterogeneity in managers' orientations and perceptions on strategic marketing management in foreign markets. Journal of Marketing Theory and Practice, 19(2), 187-204. doi:10.2753/mtp1069-6679190205
Navarro-García, A., Rondán-Cataluña, F. J., \& Acedo-González, F. J. (2013). The importance of an export-oriented culture for export performance. European Journal of International Management, 7(3), 254277. doi:10.1504/ejim.2013.054325

Onkelinx, J., Manolova, T. S., \& Edelman, L. F. (2012). The role of employee human capital in the accelerated internationalization of SMEs: Empirical evidence from Belgium. Frontiers of Entrepreneurship Research, 32(16), 1-15.

Przybylska, K. (2013). The influence of psychic distance on the internationalization of Polish born globals. Transformations in Business \& Economics, 12(2B), 525-537

Reinhard, M., Strobel T., \& Vieweg, H-G . (2013). Study on the competitiveness of the electrical and electronic engineering industry. Framework contract "Industrial competitiveness and market performance”, ECSIP consortium, European Commission, Directorate-General for Enterprise and Industry.

Rondan-Cataluna, F. J., Sanchez-Franco, M. J., \& Villarejo-Ramos, A. F. (2010). Searching for latent class segments in technological services. Service Industries Journal, 30(6), 831-849. doi:10.1080/02642060701849873

Rose, G. M., \& Shoham, A. (2002). Export performance and market orientation: Establishing an empirical link. Journal of Business Research, 55(3), 217-225. doi:10.1016/s0148-2963(00)00139-9

Ruppenthal, T., \& Bausch, A. (2009). Research on export performance over the past 10 years: A narrative review. European Journal of International Management, 3(3), 328-364. doi:10.1504/ejim.2009.026995

Saleh, M. A., Ali, M. Y., \& Mavondo, F. T. (2014). Drivers of importer trust and commitment: Evidence from a developing country. Journal of Business Research, 67(12), 2523-2530.

Schwartz, S. H. (1992). Universals in the content and structure of values: Theoretical advances and empirical tests in 20 countries. Advances in Experimental Social Psychology, 25, 1-65. doi:10.1016/Soo652601(08)60281-6

Schwartz, S. H. (2009). Draft users manual: Proper use of the Schwarz value survey, version 14. Auckland: Centre for Cross Cultural Comparisons.

Schwartz, S. H., \& Bardi, A. (2001). Value hierarchies across cultures: Taking a similarities perspective. Journal of Cross-Cultural Psychology, 32(3), 268-290. doi:10.1177/0022022101032003002

Schwartz, S. H., Cieciuch, J., Vecchione, M., Davidov, E., Fischer, R., Beierlein, C., ... Konty, M. (2012). Refining the theory of basic individual values. Journal of Personality and Social Psychology, 103(4), 663-688. doi:10.1037/aoo29393

Schweizer, R. (2012, June). The internationalization process of SMEs: A muddling-through process. Journal of Business Research, 65(6), 745-751. doi:10.1016/j.jbusres.2010.12.011

Soriano, D., \& Castrogiovanni, G. (2012). The impact of education, experience and inner circle advisors on SME performance: Insights from a study of public development centers. Small Business Economics, 38(3), 333-349. doi:10.1007/s11187-010-9278-3

Sousa, C. M. P., Martínez-López, F. J., \& Coelho, F. (2008). The determinants of export performance: A review of the research in the literature between 1998 and 2005. International Journal of Management Reviews, 10(4), 343-374. doi:10.1111/j.1468-2370.2008.00232.x 
Sousa, C. M. P., Ruzo, E., \& Losada, F. (2010). The key role of managers' values in exporting: Influence on customer responsiveness and export performance. Journal of International Marketing, 18(2), 1-19. doi:10.1509/jimk.18.2.1

Torre, E. Della, \& Solari, L. (2013). High-performance work systems and the change management process in medium-sized firms. International Journal of Human Resource Management, 24(13), 2583-2607. doi: 10.1080/09585192.2012.744337

Ural, T. (2009). The effects of relationship quality on export performance: A classification of small and medium-sized Turkish exporting fir$\mathrm{ms}$ operating in single export-market ventures. European Journal of Marketing, 43(1-2), 139-168. doi:10.1108/03090560910923274
Wagner, R., \& Paton, R. A. (2014). Strategic toolkits: Seniority, usage and performance in the German SME machinery and equipment sector. International Journal of Human Resource Management, 25(4), 475-499. doi:10.1080/09585192.2013.792863

White, J. C., Varadarajan, P. R., \& Dacin, P. A. (2003). Market situation interpretation and response:The role of cognitive style, organizational culture, and information use. Journal of Marketing, 67(3), 63-79. doi:10.1509/jmkg.67.3.63.18654

Zou, S., \& Cavusgil, S. T. (2002). The GMS: A broad conceptualization of global marketing strategy and its effect on firm performance. Journal of Marketing, 66(4), 40-56. doi:10.1509/ jmkg.66.4.40.18519 